\title{
Three cases of bronchial stump aspergillosis: unusual clinical presentations and beneficial effect of oral itraconazole
}

\author{
M. Noppen*, I. Claes*, B. Maillet**, M. Meysman*, I. Monsieur*, W. Vincken*
}

Three cases of bronchial stump aspergillosis: unusual clinical presentations and beneficial effect of oral itraconazole. M. Noppen, I. Claes, B. Maillet, M. Meysman, I. Monsieur, W. Vincken. CERS Journals Ltd 1995.

ABSTRACT: Bronchial stump aspergillosis (BSA), i.e. Aspergillus infection of bronchial granulation tissue surrounding endobronchial suture threads, is a very rare variant of localized suppurative bronchial Aspergillus infection. The majority of reported cases have occurred within one year after lung surgery.

We present three more patients, in whom BSA occurred very late $(4.5,6$ and 7 yrs) after pulmonary resection. Other unusual features were: complete absence of symptoms in one patient, and simultaneous occurrence of aspergilloma in another. Removal of the endobronchial suture probably constitutes the key therapy for BSA. In all three of our patients oral itraconazole resulted in clinical, histological and microbiological improvement.

In conclusion, BSA should be considered in the differential diagnosis of haemoptysis occurring up to 7 yrs after lung surgery, although an asymptomatic presentation is possible. Furthermore, BSA can be associated with other clinical presentations of Aspergillus infection, e.g. aspergilloma. Finally, long-term oral itraconazole therapy may represent a valid alternative when removal of the suture is not feasible. Eur Respir J., 1995, 8, 477-480.
*Respiratory Division and **Dept of Pathology, Academic Hospital, University of Brussels, Brussels, Belgium.

Correspondence: M. Noppen

Respiratory Division

Academic Hospital AZ-VUB

University of Brussels

101 Laarbeeklaan

B-1090 Brussels

Belgium

Keywords: Aspergillus infection bronchial stump aspergillosis itraconazole

Received: March 221994

Accepted after revision October 181994
Aspergillus can cause a wide variety of tracheobronchial and pulmonary disorders, depending upon the patient's local and systemic immune status and alterations in host tissues $[1,2]$. In view of the expanding spectrum of different clinical presentations of Aspergillus infection during the last decade, classification of tracheobronchial and pulmonary aspergillosis syndromes has become increasingly complicated [3]. Two or more entities may coexist, or there may be progression from one to another [1].

In some patients, Aspergillus infection is limited to the tracheal or bronchial mucosa: distinct clinical entities are saprophytic tracheobronchial aspergillosis (including mucoid bronchial impaction and obstructing bronchial aspergillosis); allergic aspergillosis (including allergic bronchopulmonary aspergillosis and bronchocentric granulomatosis); and invasive tracheobronchial aspergillosis (including Aspergillus (tracheo)bronchitis, ulcerative Aspergillus bronchitis and pseudomembranous Aspergillus tracheobronchitis) [4, 5]. Bronchial stump aspergillosis (BSA), i.e. Aspergillus infection of bronchial granulation tissue surrounding endobronchial sutures after pulmonary resection, is another (rare) form of tracheobronchial aspergillosis. Only 17 cases have so far been reported in the literature $[6,7]$. In the nine cases reported by SAWASAKI et al. [6] in 1969, BSA usually occurred 6-12 months after surgery. In only one case, BSA occurred 38 months after the intervention. In the one case reported by RoIG et al. [7], BSA occurred 4 yrs after lobectomy. The other 7 cases of BSA were published in the German literature (quoted in [6]).

We present three more cases of BSA, occurring 4.5, 7 and 6 yrs, respectively, after pulmonary resection for bronchogenic carcinoma, by far the longest latency periods so far reported.

\section{Case No. 1}

Poorly differentiated squamous cell carcinoma of the left lower lobe (stage T2N0M0) was diagnosed in 1987 in a 61 year old woman with atopic asthma. A left lower lobectomy was performed. Tumour cells were found at the cut bronchial surface, and postoperative radiotherapy (55 Gy in three sessions) was applied. The follow-up was uneventful.

The patient was then hospitalized in November 1991 for an exacerbation of asthma (for which she was not taking inhaled or oral corticosteroid therapy), which responded promptly to therapy with inhaled bronchodilators and intravenous corticosteroids. Chest roentgenography showed postlobectomy changes. During this hospitalization, a fibreoptic bronchoscopy was performed to exclude tumour recurrence. There was no evidence 


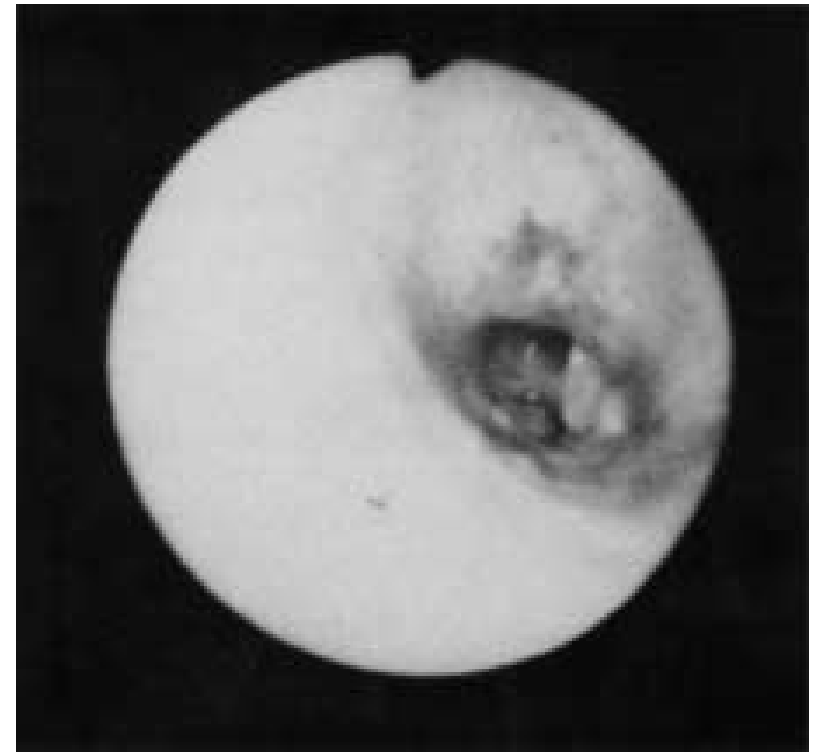

Fig. 1. - Endoscopic view of the amputation stump, showing a white "necrotic tissue" around the suture thread, and inflammation of the surrounding bronchial mucosa.

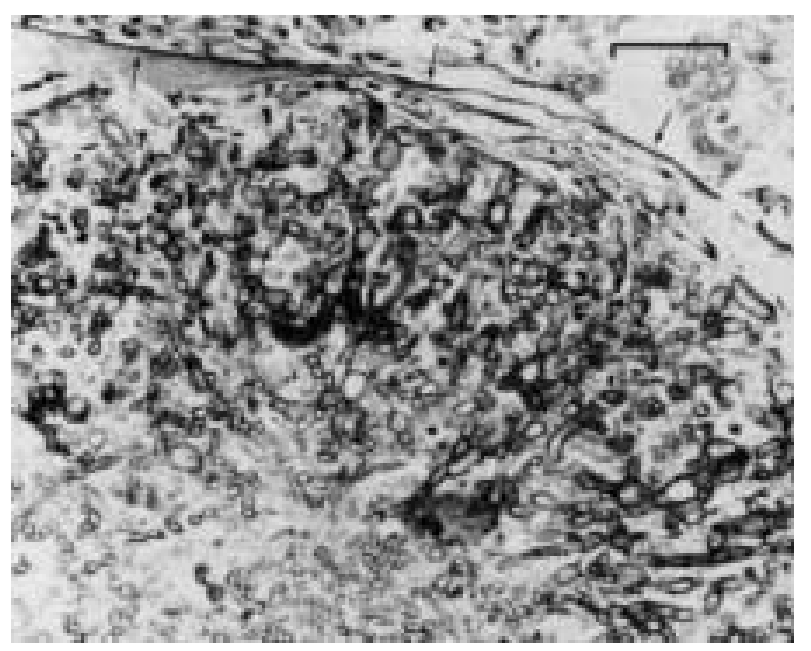

Fig. 2. - Bronchial mucosa infiltrated by Aspergillus hyphae. The basal membrane is still preserved (arrows). (Periodic-acid Schiff (PAS), $\times 125$; internal scale marker $=0.2 \mathrm{~mm}$ ).

of tumour recurrence, but the visible silk suture wires of the bronchial stump were embedded in white necrotic tissue (fig. 1). Biopsies revealed an ulcerated bronchial mucosa, extensively invaded by hyphae and fungal spores (fig. 2). Cultures of the bronchial washings yielded Aspergillus fumigatus. There were no immediate or delayed skin reactions to Aspergillus spp., and there were no precipitating antibodies to Aspergillus spp. Total immunoglobulin $\mathrm{E}$ (IgE) level was $26 \mathrm{kU} \cdot l^{-1}$ : specific IgE (radio allergosorbent test (RAST)) for Aspergillus spp. was negative. With the exception of a newly discovered type II diabetes mellitus, no predisposing risk factors for opportunistic Aspergillus infection were present.

Since removal of suture thread from the stump was

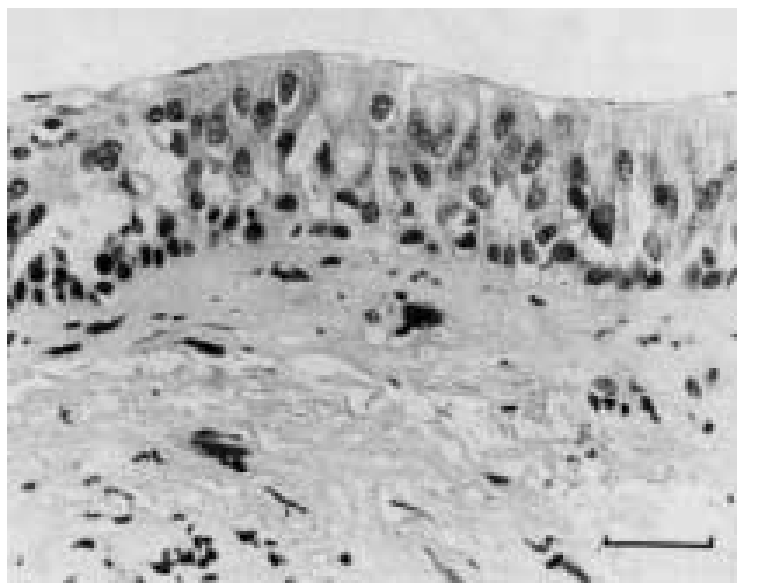

Fig. 3. - Tissue from the same patient as in figures 1 and 2, after treatment with itraconazole. The respiratory epithelium is infiltrated by a few rare lymphocytes. There is a slight thickening of the basal membrane, and the deeper layers show fibrosis and a scarce inflammatory infiltrate. (Periodic-acid Schiff $(\mathrm{PAS}), \times 250$; internal scale marker $=0.1 \mathrm{~mm})$.

refused, oral itraconazole was administered (200 mg b.i.d.). One month later, bronchoscopy showed a normal mucosa at the bronchial stump. Biopsies (fig. 3) and cultures for Aspergillus remained negative, and itraconazole was discontinued. In December 1992, the patient was readmitted for dyspnoea, cough and low grade fever. A bronchopneumonia of the right lower lobe was diagnosed, and initially treated with cefuroxime $1.5 \mathrm{~g}$ t.i.d. i.v. Repeated cultures of sputum and blood were negative. Respiratory insufficiency gradually developed, and the patient was transferred to the intensive care unit (ICU) for mechanical ventilation. Sputum cultures yielded Pseudomonas aeruginosa and methicillin-resistant Staphylococcus aureus, as well as some colonies of Aspergillus fumigatus. On bronchoscopy, the amputation stump appeared normal. Mucosal biopsies were negative for Aspergillus. The patient died three weeks later due to septic shock, with multi-organ failure. No autopsy was performed.

\section{Case No. 2}

A right pneumectomy for bronchogenic squamous cell carcinoma was performed in 1985 in an otherwise healthy 58 year old man (stage T2N0M0). No postoperative radiotherapy was given.

Follow-up was uneventful, until May 1992, when occasional expectoration of bloody sputum occurred. There were no other complaints or symptoms.

Physical examination was completely normal, except for absent breath sounds and dullness at the right hemithorax. The chest roentgenography revealed a normal postpneumectomy status. Fibreoptic bronchoscopy showed haemorrhagic granulation on the stump, around the visible silk suture wire. Mucosal biopsies revealed abundant hyphae and fungal spores, and a fungal and lymphoplasmocytic inflammatory infiltration in the mucosa, without evidence of tumour recurrence. Cultures of the bronchial aspirate yielded Aspergillus fumigatus. Oral itraconazole was given for two months, $200 \mathrm{mg}$ b.i.d. 
Within one week, the haemoptysis disappeared. Followup bronchoscopy after two months showed a normal aspect of the amputation stump. Mucosal biopsies and aspirates were negative for Aspergillus, and itroconazole was discontinued.

Six months later, haemoptysis reccurred. Bronchoscopy revealed recurrence of bronchial stump inflammation, and Aspergillus fumigatus was again cultured from bronchial aspirate. Complete mechanical removal of the suture wire was not possible. Therefore, the visible part of the wire was vapourized with the neodymium: yttrium aluminium garnet (Nd:YAG) laser, and itraconazole was restarted. Bronchoscopic findings after two months had returned to normal. Biopsies and cultures were negative for Aspergillus, and itraconazole was discontinued. There has been no recurrences at follow-up so far.

\section{Case No. 3}

Poorly differentiated large cell carcinoma of the left lung apex with sulcus superior invasion (Claude Bernard Horner syndrome) stage T3N0M0, was diagnosed in September 1986 in a 39 year old man. A left upper lobectomy and partial resection of the plexus was performed. Postoperative radiotherapy (55 Gy) was applied.

Follow-up was normal, except for the development of radiation fibrosis in the residual left upper lung region in 1989.

In September 1992, recurrent episodes of haemoptysis appeared, without any other signs or symptoms. Physical examination was strictly normal. Chest roentgenography revealed a $5 \mathrm{~cm}$ diameter lesion at the left apex. Computed tomography (CT) revealed a pediculated balllike structure, mobile at different patient positions, and with an air-menicus sign, highly suspect for mycetoma in the postlobectomy cavity. Bronchoscopy showed haemorrhagic inflammation and a white necrotic coat at the left upper lobe amputation stump, around the visible silk suture wire. Mucosal biopsies revealed a nonspecific inflammatory infiltration and fungal invasion of the mucosa, as well as abundant superficial hyphae. There were no signs of tumour recurrence. Cultures of the bronchial washings were positive for Aspergillus fumigatus. Oral itraconazole was administered (100 $\mathrm{mg}$ t.i.d.). A followup bronchoscopy one month later showed a normal aspect of the amputation stump, and biopsies and cultures had become negative for Aspergillus fumigatus. Itraconazole was continued in view of the presence of aspergilloma.

Three months later, the patient was readmitted after a single episode of massive haemoptysis (approximately $500 \mathrm{ml}$ ), which ceased spontaneously. Bronchial washings were again positive for Aspergillus fumigatus, but there was no bronchoscopic or histological recurrence of BSA. Itraconazole was continued. Other therapeutic interventions, such as endoscopic suture wire removal, bronchial arterial embolization, or surgery were all refused by the patient.

An 8 month follow-up period was uneventful. There was no recurrence of haemoptysis. A repeat bronchoscopy in August 1993 revealed a completely normal aspect of the amputation stump. Biopsies and cultures were negative for Aspergillus. CT scan still showed the presence of a fungus ball. Itraconazole treatment was continued [8, 9], until May 1994. There have so far been no recurrences of $\mathrm{BSA}$.

\section{Discussion}

We report three more cases of bronchial stump aspergillosis (BSA), a rare clinical presentation of Aspergillus tracheobronchial infection.

SAWASAKI et al. [6] first reported BSA as a specific clinical entity in the English literature. In their series of nine patients, they concluded that the use of silk thread sutures in pulmonary resection were probably responsible for the development of BSA: the incidence of BSA when silk sutures were used was about $1.5 \%$, whereas no complications were encountered in 140 resections with nylon sutures. The latency period for the development of BSA ranged 6-12 months, with the exception of one patient (38 months) [6]. Another case of BSA occurring 4 yrs after lobectomy has recently been reported [7]. Prior to these reports, seven cases of BSA were published in the German literature (mentioned in [6]).

Unlike most clinical manifestations of Aspergillus tracheobronchitis, BSA generally develops in patients who are apparently not immunocompromised [6, 7]. Our first patient had atopic asthma, which was treated with $\beta_{2}$ sympathicomimetics on demand. At the time of diagnosis of BSA, she was receiving parenteral (total dose $440 \mathrm{mg}$ ) followed by oral methylprednisolone $(16 \mathrm{mg}$ once daily). Furthermore, type II diabetes mellitus was discovered. Although corticosteroids can enhance fungal invasion by various mechanisms [10-12], and although severe diabetes with refractory hyperglycaemia and ketoacidosis can predispose to fungal infections [13], it is more probable that in our patient the local airway "disturbance" by the silk suture thread was responsible for the development of BSA.

Patients with BSA usually present with cough and expectoration of putrid or bloody sputum, and occasionally of fungal material or suture thread, although one of our patients was completely asymptomatic at the time of diagnosis. Fibrebronchoscopic appearance of the bronchial stump can be normal or, more often, can show the suture thread surrounded by inflamed bronchial mucosa or granulation tissue, covered with purulent material.

Bronchial mucosal biopsies typically yield inflammatory and necrotic areas, with infiltration by abundant hyphae showing the morphological features of Aspergillus. Cultures are usually positive for Aspergillus.

In the published cases of BSA, no other concomitant forms of Aspergillus infection were present, although clinical overlap between, and evolution to, different Aspergillus syndromes are well-known [14]. In our third patient, aspergilloma was present in addition to BSA.

Although no comparative studies exist, removal of the silk suture probably constitutes the key therapy for BSA $[6,7]$, and should be attempted. SAWASAKi et al. [6] suggested the use of local or inhaled fungicides (iodine tincture, phenylacetic quicksilver), in addition to removal of the silk thread. 
Endobronchial sutures can usually be removed easily and safely by means of the flexible bronchoscope, using standard or alligator cup forceps [15]. Sometimes, endoscopic suture scissors [15] or Nd:YAG laser [16] are necessary for removal.

In case No. 1, bronchoscopic suture thread removal was refused by the patient because of fear of complications on the already operated lung. Treatment with oral itraconazole achieved a complete disappearance of bronchoscopic, histological and microbiological signs of BSA after one month. This experience incited the initial use of itraconazole at a similar dosage in case No. 2. After two months, all signs of BSA had disappeared. Since BSA recurred 6 months after the cessation of itraconazole, the suture thread was evaporated by means of the $\mathrm{Nd}$ :YAG laser, and itraconazole was restarted for two months. Thereafter, there was no recurrence of BSA. In case No. 3, itraconazole was started in view of the preceding results, and the presence of concomitant aspergilloma [8]. One month later, all signs of BSA had disappeared. Itraconazole treatment was continued in view of the presence of aspergilloma for a total of 20 months [8]. There has been no recurrence of BSA, in spite of the still present suture wire.

Oral itraconazole was, thus, able to suppress the infection sufficiently to alleviate symptoms in one case (No. 2 ), and return to normal the endoscopic and histological appearance of the bronchial stump mucosa in all three cases, despite retention of suture material.

In conclusion, our findings show that BSA should be considered in the differential diagnosis of haemoptysis occurring after lung resection surgery, up to 7 yrs after the intervention, although an asymptomatic presentation is possible. Furthermore, BSA can be associated with other clinical presentations of Aspergillus infection, e.g. aspergilloma.

Treatment would probably consist of removing the bronchial suture threads. If this is not feasible, oral itraconazole may represent a valid alternative. Larger series are needed to confirm these findings, and to establish the optimal dosage and duration of treatment, particularly taking into account the rather poor bioavailability of itraconazole with the present formulation.

\section{References}

1. Rohatgi PK, Rohatgi NB. Clinical spectrum of pulmonary aspergillosis. South Med J 1984; 77: 1291-1301.

2. Klein DL, Gamsu G. Thoracic manifestations of aspergillosis. AJR 1980; 134: 543-552.

3. Bodey GP, Vartivarian S. Aspergillosis. Eur J Clin Microbiol Infect Dis 1989; 8: 413-437.

4. Kramer MR, Denning DW, Marshall SE, et al. Ulcerative tracheobronchitis after lung transplantation: a new form of invasive aspergillosis. Am Rev Respir Dis 1991; 144: 552-556.

5. Clarke A, Skelton J, Fraser RS. Fungal tracheobronchitis: report of 9 cases and review of the literature. Medicine 1991; 70: 1-14.

6. Sawasaki H, Kazuo H, Yamada M, et al. Bronchial stump aspergillosis: experimental and clinical study. $J$ Thorac Cardiovasc Surg 1969; 58: 198-208.

7. Roig J, Ruiz J, Puig X, Carreras A, Morera J. Bronchial stump aspergillosis four years after lobectomy. Chest 1993; 104: 295-296.

8. Dupont B. Itraconazole therapy in aspergillosis: study in 49 patients. J Am Acad Dermatol 1990; 23: 607-614.

9. Loeuille GA, Berthou C, Hilmarsdottir I, Menard O, Sardet Frismand A. Aspergilloses traitées par l'itraconazole: analyse de 64 cas. J Mycol Méd 1993; 3: 1-13.

10. Dale DC, Petersdorf RG. Corticosteroids and infectious diseases. Med Clin North Am 1973; 57: 1277-1287.

11. Merkow LP, Epstein SM, Sidransky H, Verney E, Pardo M. The pathogenesis of experimental pulmonary aspergillosis: an ultrastructural study of alveolar macrophages after phagocytosis of A. flavus spores in vivo. Am J Pathol 1971; 62: 57-66.

12. Schaffner A, Douglas H, Brande A. Selective protein against conidia by mononuclear and against mycelia by polymorphonuclear phagocytes in resistance to Aspergillus: observations on these two lines of defense in vivo and in vitro in human and mouse phagocytes. J Clin Invest 1982; 69: 617-631.

13. Weber DJ, Rutula WA. Epidemiology of nosocomial fungus infections. Curr Top Med Mycol 1988; 2: 305-337.

14. Pennington JE. Aspergillus. In: Sarosi GA, Davies SF eds., Fungal Diseases of the Lung. New York, Raven Press Ltd, 1993; pp. 133-147.

15. Shure D. Endobronchial suture: a foreign body causing chronic cough. Chest 1991; 100: 1193-1196.

16. Unger M. Nd:YAG laser therapy for malignant and benign endobronchial obstructions. Clin Chest Med 1985; 6: 277-290. 